Research

\title{
Association between co-authorship network and scientific productivity and impact indicators in academic medical research centers: A case study in Iran \author{
and Arash Etemadi ${ }^{4}$
} \\ Reza Yousefi-Nooraie ${ }^{* 1,2}$, Marjan Akbari-Kamrani ${ }^{2}$, Robert A Hanneman ${ }^{3}$
}

Address: ${ }^{1}$ Center for Academic and Health Policy, Tehran University of Medical Sciences, Tehran, Iran, ${ }^{2}$ Students' Scientific Research Center, Tehran University of Medical Sciences, Tehran, Iran, ${ }^{3}$ Department of Sociology, University of California, Riverside, USA and ${ }^{4}$ Department of Epidemiology and Biostatistics, School of Public Health, Tehran University of Medical Sciences, Tehran, Iran

Email: Reza Yousefi-Nooraie* - ryousefi@razi.tums.ac.ir; Marjan Akbari-Kamrani - marjan_a_k@yahoo.com;

Robert A Hanneman - robert.hanneman@ucr.edu; Arash Etemadi - aetemadi@tums.ac.ir

* Corresponding author

\section{Published: 16 September 2008}

Health Research Policy and Systems 2008, 6:9 doi:10.1 186/1478-4505-6-9

This article is available from: http://www.health-policy-systems.com/content/6/1/9

(C) 2008 Yousefi-Nooraie et al; licensee BioMed Central Ltd.

This is an Open Access article distributed under the terms of the Creative Commons Attribution License (http://creativecommons.org/licenses/by/2.0), which permits unrestricted use, distribution, and reproduction in any medium, provided the original work is properly cited.
Received: 23 June 2008

Accepted: 16 September 2008

\begin{abstract}
Background: We aimed to examine the co-authorship networks in three successful Iranian academic research centers, in order to find the association between the scientific productivity and impact indicators with network features in a case study.

Methods: We searched for English articles of the three research centers. We drew co-authorship maps of each center and calculated social network measures.

Results: The collaboration networks in centers shared many structural features, including a "starlike" pattern of relations. Centers with more successful scientific profile showed denser and more cooperative networks. Key figures in each center were interviewed for their understandings of the reasons for the emergence of these patterns.

Conclusion: Star shape network structure and dependency on a single big member is a common feature observed in our case study. Scientific output measures correlate with the network structure of research centers. Network analysis seems a useful method to explore the subtle scientific contexts in research organizations.
\end{abstract}

\section{Introduction}

The process of research evaluation is of major importance for the development of health systems[1,2]. In this respect, centers within large medical schools are very important contexts for research production. Biomedical Research tends to be highly collaborative[3], and research institutions and academic centers bring researchers together in productive relationships. The quantity, quality, and creativity of the work produced in medical research centers vary. Much of the variation may well be due to the individual talent, expertise, and enterprise of the researchers. The structure of social relations, roles, and leadership, however, may also make critical differences.

Social network analysis provides a number of methods for revealing patterns of interpersonal relationships, and have frequently been applied to study collaboration through co-authorship networks [3-5]. Network analyses are 
becoming an important part in the growing body of research on social capital $[6,7]$.

In the present study, we examined the co-authorship networks of researchers in three high profile Iranian research centers, and aimed to use it as a case study to investigate the association between the overall scientific productivity and impact indicators with collaboration network features.

\section{Methods}

\section{- Co-authorship networks}

Data were collected on co-authorship of papers published in English by members of three research centers: Endocrinology and Metabolism Research Center (EMRC), Digestive Diseases Research Center (DDRC) and Pharmaceutical Sciences Research Center (PSRC); which were the first three highest ranked research centers in Tehran University of Medical Sciences respectively and each has been at least once the top Iranian research center in the past 5 years [8]. These centers were selected because they had sufficient and similar number of articles to be analyzed. We searched the ISI Web of Science database for all articles, published since the establishment of each center until September 2006, referring to the center's name at the correspondence address. Because there was not any explicit membership definition for research centers, and there were a lot of people collaborating occasionally with the centers (in the form of theses or part time research), we defined the research center membership as having at least two articles affiliated by the corresponding center. In addition, the scientific secretaries of each center reviewed the lists to confirm the membership of selected authors.

We generated a table for each research center, with the rows formed by the list of members and the columns by the articles. A "co-authorship matrix" showing the number of collaborations between each pair of members was generated. The information regarding authors who were not considered as the members of centers was gathered as a single "outsiders" row.

\section{- Scientific productivity and impact indicators}

We used ISI Web of Knowledge database to obtain following information for each article: the impact factors of the publishing journals (of the years of publication of the articles), and the number of citations received during two years after publication. We also obtained some other scientific productivity measures from an evaluation project conducted by the deputy for research of Tehran University of Medical Sciences in 2005-6[8]. These measures were the number of doctoral/master's theses, multi-centric research projects, obtained grants from external funding bodies, and seminars held by each center.

\section{- Analysis}

The impact factors, the number of received citations and other scientific productivity measures were compared between three centers, and post hoc pairwise comparisons were performed when needed. Ucinet[9] and Pajek[10] software programs were used for network analysis of each center.

We calculated basic network descriptive measures and the average number of collaborators per author, regardless of the volume of production. To examine this, the data were dichotomized (i.e. if two actors co-authored any papers they were coded one) making a "collaboration matrix". Analyses of the structure of collaboration are based on the dichotomized data, showing whether two persons had ever collaborated.

Because of the considerable skewness of network measures, non-parametric measures of central tendency and dispersion were used. These measures were compared between the centers using Kruskall-Wallis test.

Degree centralization, and betweenness centralization measures were used to investigate whether collaboration is equally distributed across the researchers in each center, or there is a tendency for some actors to be more "central" to the web of collaboration [11]. At one extreme, a single individual or "star" may dominate - having far more collaborative connections than others, and acting as a broker or patron. At the other extreme, while there may be inequality, members are more able to form alternative collaborations, and are less likely to be dominated by the central elite. Star-shaped networks may be less productive than those with denser and more horizontal connections $[12,13]$. The degree centralization measure examines the inequality in the distribution of collaborators across researchers, varying between $0 \%$ to $100 \%$, which shows the similarity of the network to star-shaped pattern[11]. The betweenness centralization measure examines the extent to which the shortest paths between pairs of actors in the collaboration network pass through a third actor [14]. To the extent that actors are "between" pairs of others, they may act as coordinators and brokers, and may gain status and power.

A frequent finding in collaboration networks is that they resemble "Small worlds"[15], particularly larger networks. That is, they have considerable clustering (clustering coefficient; which measures the probability that two of a scientist's coauthors have themselves coauthored a paper) combined with relatively low average geodesic distances (i.e. each actor in the network is "close" to all other actors). Creative scientific research on the one hand requires the close support of a community of peers who are expert in closely related areas, and on the other hand, 
needs getting access to the diverse sources of knowledge in other specialties. The simultaneous presence of dense local clustering with short social network distance to diverse others [15] is a characteristic feature of facilitated knowledge flow inside networks[16,17].

One approach to examine the structure of collaboration (social positions) is called "structural block modeling"[11]. In this approach, we group together actors who are similar in terms of the pattern of ties that they have to all other actors. The resulting simplified pattern shows who belong to which "cluster," and which clusters collaborate, or do not, with one another. We used a five or six block model to investigate the structural equivalence of the centers, determined by the overall goodness of fit.

Using different centralization measures, three most central actors were identified in each network. We interviewed some active members of each center and discussed the potential reasons for importance of core people, the similarities in the roles of members of clusters, and the relationships between the observed clustering and formal and informal categorizations in the centers.

\section{Results}

- Scientific productivity and impact indicators

The scientific activity measures of three centers are shown in table 1 . The mean journal impact factor of DDRC was significantly more than two others (p: 0.0001). EMRC articles received the lowest citations among three centers (p: 0.003). The percentages of multi-centric projects and obtained grants from external bodies in PSRC were significantly less than other centers.

\section{- Co-authorship networks}

Some descriptive statistics of three centers and their comparisons are shown in table 2. The mean papers per author and mean authors per paper in PSRC were significantly lower than two other centers.

\section{Degree distribution}

There were notable differences in the absolute number of co-authorships between centers. All three centers displayed significant positive skewness in the distribution of co-authorship (figure 1). It was substantially greater in the
DDRC and EMRC than in the PSRC. The overall productivity (median and mean papers per author) was also greatest in the former two centers.

The overall collaboration (regardless of the volume of production) in DDRC and EMRC was higher than PSRC; moreover, the distribution of the volume of production was more unequal than the distribution of collaboration.

\section{Centralization}

All three centers displayed high degree centralization, though it was less in PSRC. The DDRC and EMRC had a more characteristic single "star" pattern. The PSRC is characterized by two actors who were somewhat more involved in collaborations than the others, but similar to one another. The betweenness centralization was greatest at PSRC.

\section{Small world phenomenon}

As shown in table 2, all 3 centers displayed quite high levels of clustering. The average distance between members of the center, however, was much greater in PSRC (2.3) than DDRC or EMRC (both 1.6); This greater distance suggests that, in PSRC there were less actors who connect multiple clusters, showing a less typical "small-world".

\section{Collaboration with outsiders}

The DDRC was distinguished by the very high rate of collaboration with outsiders (non-members). The relative amount of collaboration "inside" and "outside" the organization was about the same for DDRC (14/21) and PSRC members (5/5.5) while EMRC members (10/6) showed a tendency toward relatively more "internal" activity.

Spearman's correlation coefficients for the association between the amount of inside and outside collaborations are shown in table 2. It was notably pronounced at DDRC which suggests it was the more central people who were most likely to have outside connections. This was notably less in PSRC, where outside ties are the same across all levels of the internal hierarchy.

Table I: Scientific productivity and impact indicators of three centers

\begin{tabular}{|c|c|c|c|c|}
\hline & DDRC & EMRC & PSRC & p-value \\
\hline Impact factor, mean (SD) & $2.7 I(1.4)$ & I.37(0.99) & I.77(0.77) & 0.0001 \\
\hline Received citations, median (interquartile range) & 2(4) & $0(1.25)$ & $2(4.25)$ & 0.003 \\
\hline Doctoral/master's theses (percentage of total running research projects) & $30(43 \%)$ & $17(33 \%)$ & $7(30 \%)$ & 0.4 \\
\hline Multi-centric projects (percentage of total running research projects) & $32(46 \%)$ & $18(35 \%)$ & $\mathrm{I}(4 \%)$ & 0.001 \\
\hline obtained grants from external funding bodies(percentage of total running research projects) & $18(26 \%)$ & $12(24 \%)$ & $\mathrm{I}(4 \%)$ & 0.09 \\
\hline seminars held & 3 & 1 & 0 & - \\
\hline
\end{tabular}


Table 2: Bibliometric statistics and network measures in three centers

\begin{tabular}{|c|c|c|c|c|}
\hline & DDRC & EMRC & PSRC & pvalue \\
\hline Total papers & 48 & 50 & 54 & - \\
\hline Total authors (size of network) & 39 & 33 & 36 & - \\
\hline Papers per author (median(IQ range)) & $4(4)$ & $4(4)$ & $2(2.75)$ & $P=0.006$ \\
\hline Maximum papers per author & 42 & 43 & 22 & - \\
\hline Mean authors per paper & $5(3)$ & $4(2.2)$ & $2.7(1.3)$ & $\mathrm{p}<0.0001$ \\
\hline Maximum authors per paper & 12 & II & 6 & - \\
\hline
\end{tabular}

\section{Co-authorship network}

\begin{tabular}{|c|c|c|c|c|}
\hline Average co-authorship (median(IQ range)) & $23(35)$ & $18(15)$ & $6.5(7.5)$ & $P<0.0001$ \\
\hline Collaborators per author (median(IQ range)) & $14(9)$ & $10(7.5)$ & $5(3)$ & $\mathrm{p}<0.0001$ \\
\hline
\end{tabular}

\begin{tabular}{|c|c|c|c|c|}
\hline Network centralization & & & & \\
\hline Degree centralization & $61.5 \%$ & $63.8 \%$ & $50.6 \%$ & - \\
\hline Betweenness centralization & $15.6 \%$ & $27.7 \%$ & $57.2 \%$ & - \\
\hline \multicolumn{5}{|l|}{ Small world phenomenon } \\
\hline Average density & .417 & .369 & .151 & - \\
\hline Clustering coefficient & .729 & .717 & .735 & - \\
\hline Mean geodesic distance & 1.6 & 1.6 & 2.3 & - \\
\hline \multicolumn{5}{|l|}{ Outsiders } \\
\hline Outsiders per author (median(IQ range)) & $21(26)$ & $6(10)$ & $5.5(5)$ & $P<0.0001$ \\
\hline Spearman's correlation of inside and outside collaborations & $0.8(p<0.001)$ & $0.49(\mathrm{p}<0.00 \mathrm{I})$ & $0.32(p>0.05)$ & - \\
\hline
\end{tabular}

Structurally equivalent groups

The groups of "similar" actors, defined by structural block modeling, are shown with colors in figures $2 \mathrm{~A}, \mathrm{~B}$, and 2C. Data on central actors (defined by three centralization measures) in each center are shown in table 3.

\section{- DDRC}

One plausible block model divided the actors into five groups (figure 2A). This model fitted the "ideal" pattern imperfectly (R-square $=.425)$. Actors AA (head of the center), EE and J were defined as the most central people. We discussed the observed pattern of network with actors $\mathrm{Z}$ and $\mathrm{HH}$. Both stated that, the three central actors played the most influential roles in promoting research projects and connecting the members. One reason for actor AA to be central was his position as the head of the center, and for actor EE as the head of the lab. Two interviewees considered actor J as central actor, mainly because of his personal abilities as a facilitator and promoter. The circles with vertical lines (the Cancer research team) are the current members of the oldest group of the center which many other groups stemmed from it. This group played an important role in internal and external communications of DDRC.

\section{- EMRC}

A five-group block model provided a plausible summary of the data for EMRC (R-square = .491). Structurally, the pattern of collaboration at EMRC was similar to that of DDRC. Compared with DDRC, local clusters were tighter, and more separated from one another (figure $2 \mathrm{~B}$ ). The role of the "core" in integrating the center was greater. Actors FF (head of the center), KK and Z were identified as most central. The observed network was discussed with actors JJ and J. Both stated that the three most central people possessed the key positions in the center (head, deputy and secretary respectively). Two most influential and relatively independent groups in EMRC were the Osteoporosis research team (mainly whites) and the Lipid research team (Vertical lines). FF, JJ and KK were the oldest members and founders of EMRC, and JJ is currently the head of the Osteoporosis research team. J is the head of Evidence based medicine research team, an independent entity working under EMRC's umbrella; therefore he showed high betweenness despite a non-central role.

\section{- PSRC}

A six group model was used, because one actor was unique in forming a "group" (figure 2C, circle with vertical lines). The overall fit of the model was moderate (R- 

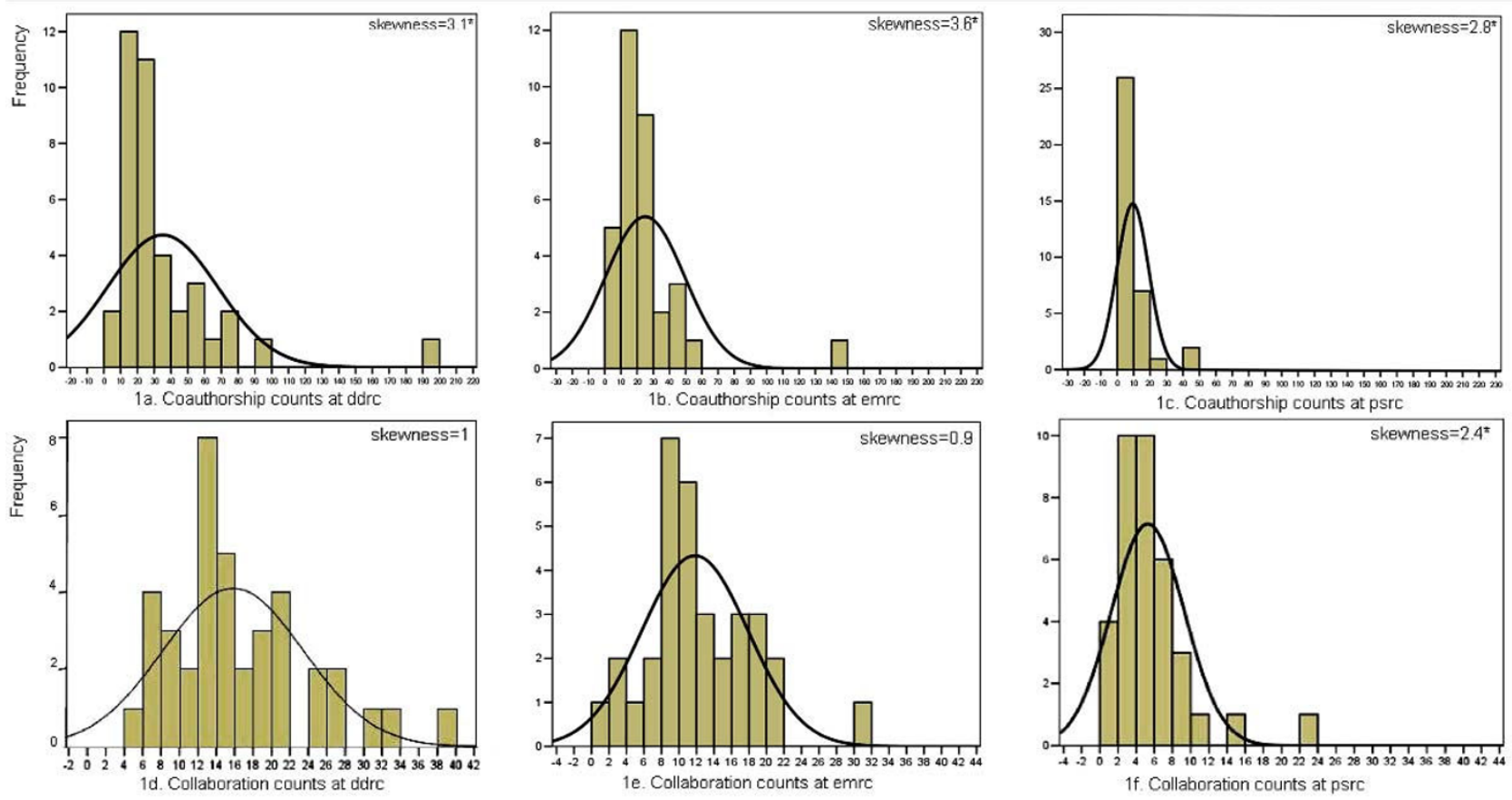

Figure I

Co-authorship and collaboration count histograms in three centers with the associated normal curves and skewness measures.

squared $=0.456)$. There was a notable tendency towards thinner ties between some groups, suggesting a lower cohesion in PSRC.

There were two tight clusters (oblique lines and grey), which did hot have strong ties to one-another (figure 2C).
Actors L (head of the center) and B were most central. The observed pattern was discussed with actors B and $\mathrm{H}$. Both explained it mainly as a result of the existence of two distinct research disciplines of Clinical Pharmacology (oblique lines) and Medicinal Chemistry (grey and white) in the center, with different research methodologies and

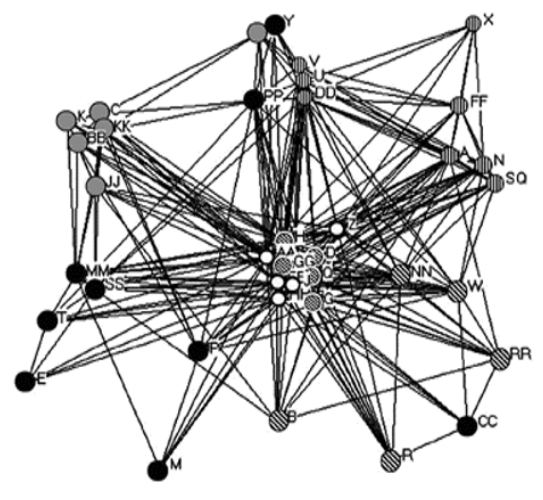

$2 A$

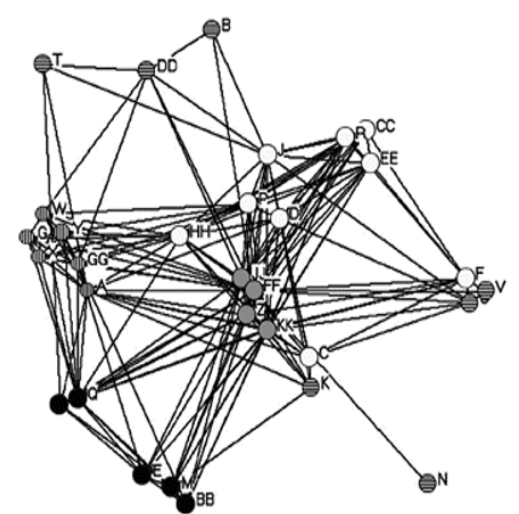

2B

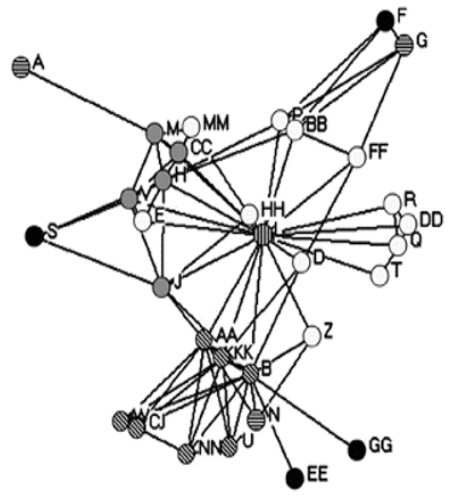

$2 \mathrm{C}$

Figure 2

Collaboration network at DDRC (2A), EMRC (2B) and PSRC (2C) with structurally equivalent blocks colored. 
Table 3: Central actors in each center based on three centrality measures

\begin{tabular}{|c|c|c|c|c|c|c|}
\hline & \multicolumn{2}{|c|}{ Degree centrality } & \multicolumn{2}{|c|}{ Betweenness centrality } & \multicolumn{2}{|c|}{ Eigenvector centrality } \\
\hline & Actor & Centrality & Actor & Centrality & Actor & Centrality \\
\hline \multicolumn{7}{|l|}{ DDRC } \\
\hline & AA & 38 & AA & 118 & AA & .289 \\
\hline & $\mathrm{EE}$ & 32 & $\mathrm{EE}$ & 56 & $\mathrm{EE}$ & .264 \\
\hline & $J$ & 30 & J & 38 & $J$ & .259 \\
\hline \multicolumn{7}{|l|}{ EMRC } \\
\hline & $\mathrm{FF}$ & 31 & $\mathrm{FF}$ & 143 & $\mathrm{FF}$ & .334 \\
\hline & KK & 21 & J & 22 & KK & .273 \\
\hline & Z & 20 & $\mathrm{~A}, \mathrm{KK}$ & 22 & Z & .267 \\
\hline \multicolumn{7}{|l|}{ PSRC } \\
\hline & $\mathrm{L}$ & 22 & L & 351 & L & .428 \\
\hline & B & 14 & B & 129 & $B$ & .346 \\
\hline & & & AA & 49 & AA & .303 \\
\hline
\end{tabular}

very rare common projects, and therefore infrequent relationships. Consequently, PSRC consisted of two star shaped independent networks. Actor $\mathrm{B}$ is the manager of the Clinical Pharmacology team.

\section{Discussion}

The network structures associated with the scientific productivity and impact indicators in our case study. Centers with denser, more decentralized, and more open to outside connections networks showed better scientific outputs. Even though, all three centers shared many network features, demonstrating common infrastructural characteristics and obstacles of research organizations in developing countries.

All three networks were structurally star shaped and centralized. The central actors consisted of similarly the heads of the centers. This is a phenomenon which is seen in many team works in developing world. A single charismatic person, who initiates, maintains and pushes the organization. Two main reasons for this limited autonomy of members and involvement of the big person on almost all operations may be the lack of professional trained researchers who are able to manage the projects independently, and the instability of the system, in which only the organizations with support from a powerful actor are able to survive. This dependency could threat the sustainability of research centers and diminish the knowledge flow between members. Rulke and Galaskiewicz showed that hierarchical structures are not efficient for complicated problems while more intense and decentralized networks provide the maximal knowledge flow between actors [12]. In addition, the central actors in three centers possess other demanding responsibilities which reduce their ability to monitor all routines in the centers. The occurrence of such betweenness in few people in a network makes it highly susceptible to break down after the removal of such actors[18].

One explanation for the observed difference in the average authors per paper in three centers is the higher likelihood of guest authorship, because of the presence of more prestigious and socially important members in some centers. Guest authorship is prevalent in scientific papers[19]. As showed by Bhopal et al. in many situations it happens unintentionally, and the powerful people may be unaware of being included as author by their novice peers[20]. In many Iranian research centers research is performed by novice general practitioners or clinical faculty members who work as part time amateur researchers. This lack of professionalism may enhance such phenomenon.

In our case study one network (DDRC) showed denser and more distributed network measures (highest average co-authorship, average collaboration, density, and least betweenness centralization, and mean geodesic distance). The scientific output measures were consistent with the network findings; depicting that the production of more cited research (a surrogate of higher quality research) seems to be associated with the inter-actor associations and knowledge flow inside research organizations.

DDRC members had more collaboration with outsiders, which made the center more open, and facilitated the potential of idea exchange and conducting interdisciplinary and multi-centric projects. Research centers are communities of a sort. At one extreme, they may be very "open" where members are equally likely to have ties outside, as inside the center. At the other extreme, they may be quite "closed". Some degree of closure probably contributes to "identity" and a "sense of mission". Too much closure may contribute to isolation from the larger world 
of science. It also may matter where the openness occurs. In many centers, the central figures and leaders act as the primary liaison between the center and the larger community; in other centers, many individuals - and not always central individuals - may have strong external ties. The former pattern may contribute to cohesion and collaboration; the latter pattern may contribute to factionalization and lower social density, which was seen more apparently in PSRC network, where articles received relatively high citations while network showed lowest productivity measures comparing to other centers. One reason for PSRC's high median citations per article in spite of lower productivity indicators and network measures, is the attractiveness of pharmacological research in comparison to clinical research, as shown by other scientometric studies [21].

An archetypical small-world network as stated by Moody "will have many distinct clusters, connected to each other by a small number of links" [22]. This clustering preserves the independence and autonomy of the clusters, while the paths of the knowledge exchange among them are open. DDRC and EMRC showed to be more typical small worlds. Several studies have demonstrated that the information diffusion and knowledge exchange are faster in small worlds $[15,23]$. Coexistence of dense and weak relationships in small worlds showed to enhance innovation. Because the dense and clustered relationships raise trust and cooperation, while ties to other clusters bring new information to the cluster[24,25].

PSRC had lowest mean authors per paper, mean papers per author, density, and highest mean geodesic distance between members, and betweenness centralization. Lower mean authors per paper means the involvement of smaller number of researchers in common projects, and lower potential for team working. This measure highly depends on the research discipline. Newman showed that the average authors per paper is 3.75 in biological sciences, 2.53 in physics and 1.45 in mathematics. He explained this difference because of involvement of large groups of field and laboratory scientists in biological research in comparison to more theoretical and individual nature of mathematical sciences [26].

Taking a closer look at networks and inter-relationships between entities as an important aspect of sustainable capacity development [27] is essential for developing countries. This warrants the assessment and development of interpersonal and inter-institutional networks to set up durable enhancing frameworks for innovation [28]. Network analysis methods seem effective and meaningful.

Our study is limited in some ways. Co-authorship relationship seems a rational- but stringent- definition of sci- entific collaboration. Knowing each other is a prerequirement for writing a paper together [3]. Co-authorship networks do not absolutely reflect the scientific collaboration, because many researchers may know and influence each other but never collaborate in writing a paper. In addition, several factors may interact on coauthorship events; many of which are not purely scientific. We limited our study to the published English language articles containing the center name in the ISI address field. This approach omits the Persian language articles and those that are written by the members but do not include the center's name in the address field. We also used a tough method for defining the membership. This may overestimate the number of outsiders, because of the presence of part time members of research centers who were considered outsiders in our study.

The scientific productivity and impact of research organizations was associated with their network structure in our case study. Social network analysis seems a useful method to explore and visualize the subtle scientific norms and customs in research centers and hidden structural and functional sources of their success or failure. Qualitative approaches to obtain the network members' views regarding network analysis outputs lead in more meaningful and less mechanical interpretations of the hidden structure.

\section{Competing interests}

The authors are not members of any of the research centers under study. No conflicts of interests are declared. AE is working as an associate editor in a peer reviewed journal which is founded by the dean of one research center under study.

\section{Authors' contributions}

conception of the research: RYN, MAK, AE; performing the research: RYN, MAK; data analysis and interpretation of findings: RYN, MAK, RAH; preparation and reviewing of manuscript: RYN, MAK, RAH, AE

\section{Acknowledgements}

We thank Hojjat Salmasian for his contribution in generating data matrices, and Dr Arash Rashidian MD. PhD for his critical comments on the manuscript drafts. The study was supported by a grant from Tehran University of Medical Sciences.

\section{References}

I. Groneberg-Kloft B, Scutaru C, Kreiter C, Kolzow S, Fischer A, Quarcoo D: Institutional operating figures in basic and applied sciences: Scientometric analysis of quantitative output benchmarking. Health Res Policy Syst 2008, 6: 1478-4505.

2. Hanney S, Gonzalez Block M: Why national health research systems matter. Health Res Policy Syst 2008, 6:.

3. Newman M: The structure of scientific collaboration networks. Proceedings of the National Academy of Sciences of the United States of America 200I, 98:404-409. 
4. Otte E, Rousseau R: Social network analysis: a powerful strategy, also for the information sciences. Journal of Information Science 2002, 28:44I-453.

5. Kretschmer H, Aguillo I: Visibility of collaboration on the Web. Scientometrics 2004, $61: 405-426$.

6. Burt R: The contingent value of social capital. Administrative Science Quarterly 1997, 42:339-365.

7. Scott C, Hofmeyer A: Networks and social capital: a relational approach to primary healthcare reform. Health Res Policy Syst 2007, 5:9.

8. Samadi N, Alhani Z, Jadidi M, Kharaman Z: Evaluation of research activities of research centers of Tehran University of Medical Sciences (2005-2006) Tehran: Tehran University of Medical Sciences Press; 2006.

9. Borgatti S, Everett M, Freeman L: Ucinet for Windows: Software for Social Network Analysis. [6]. Harvard, MA: Analytic Technologies; 2002. Ref Type: Computer Program

10. Batagelj V, Mrvar A: Pajek program for large network analysis. [I.02]. 2000 [http://vlado.fmf.uni-li.si/pub/networks/pajek/]. Ljubjana, Slovenja, University of Ljubjana Ref Type: Computer Program

II. Hanneman R, Riddle M: Introduction to social network methods. 2005 [http://faculty.ucr.edu/ hanneman/]. Riverside, CA: University of California Ref Type: Electronic Citation

12. Rulke D, Galaskiewicz J: Distribution of Knowledge, Group Network Structure, and Group Performance. Management Science 2000, 46:6/2-625 [http://mansci.journal.informs.org/cgi/content/ abstract/46/5/612].

13. Back K: Intervention techniques: Small groups. Ann Rev Psych 1974, 25:367-388.

14. Hawe P, Webster C, Shiell A: A glossary of terms for navigating the field of social network analysis. J Epidemiol Community Health 2004, 58:97।-975.

15. Watts D: Small Worlds: The dynamics between order and randomness Princeton: Princeton University Press; 1999.

16. Watts D, Strogatz S: Collective dynamics of small world networks. Nature 1998, 393:440-442.

17. Flemming L, King C, Juda L: Small Worlds and Innovation. 2004 [http://www.bus.umich.edu/Academics/Departments/CSIB/CSIB/ Fleming 04-15-05 Seminar Paper.pdf]. Harvard Business School Ref Type: Electronic Citation

18. Holme P, Kim BJ, Yoon CN, Han SK: Attack vulnerability of complex networks. Phys Rev E 2002, 65:056I09.

19. Bennett $D$, Taylor $D$ : Unethical practices in authorship of scientific papers. Emergency Medicine 2003, I5:263-270.

20. Bhopal R, Rankin J, McColl E, Thomas L, Kaner E, Stacy R, et al: The vexed question of authorship: views of researchers in a British medical faculty. BMJ 1997, 314:1009-1012.

21. Adams J, Gurney K, Marshal S: Profiling citation impact: A new methodology. Scientometrics 2007, 72:325-344.

22. Moody J: The Structure of a Social Science Collaboration Network: Disciplinary Cohesion from 1963 to 1999. American Sociological Review 2004, 69:213-238.

23. Yamaguchi K: The structural and behavioral characteristics of the smallest-world phenomenon: minimum distance networks. Social Networks 2002, 24:161-182.

24. Cowan $\mathrm{R}$, Jonard $\mathrm{N}$ : The dynamics of collective invention. Journal of Economic Behavior and Organization 2003, 52:5I 3-532.

25. Cowan R, Jonard N: Network structure and the diffusion of knowledge. Journal of Economics Dynamics and Control 2004, 28:1557-1575.

26. Newman M: Coauthorship networks and patterns of scientific collaboration. Proceedings of the National Academy of Sciences 2004, I01:5200-5205.

27. UNDP: Scientific Capacity in Developing Countries, Postnote, No. 216 March United Kingdom: Parliamentary Office of Science and Technology; 2004.

28. Cooke J: A framework to evaluate research capacity building in health care. BMC Fam Pract 2005 2005, 6:44.
Publish with Biomed Central and every scientist can read your work free of charge

"BioMed Central will be the most significant development for disseminating the results of biomedical research in our lifetime. "

Sir Paul Nurse, Cancer Research UK

Your research papers will be:

- available free of charge to the entire biomedical community

- peer reviewed and published immediately upon acceptance

- cited in PubMed and archived on PubMed Central

- yours - you keep the copyright
BioMedcentral 